University of Wollongong

Research Online

Faculty of Science, Medicine and Health -

Papers: part A

Faculty of Science, Medicine and Health

$1-1-2005$

\title{
New informatics-based work flow paradigms in radiation oncology: the potential impact on epidemiological cancer research
}

Andrew Alexis Miller

Oncology Information Services, amiller@uow.edu.au

Follow this and additional works at: https://ro.uow.edu.au/smhpapers

Part of the Medicine and Health Sciences Commons, and the Social and Behavioral Sciences

Commons

\section{Recommended Citation}

Miller, Andrew Alexis, "New informatics-based work flow paradigms in radiation oncology: the potential impact on epidemiological cancer research" (2005). Faculty of Science, Medicine and Health - Papers: part A. 2041.

https://ro.uow.edu.au/smhpapers/2041

Research Online is the open access institutional repository for the University of Wollongong. For further information contact the UOW Library: research-pubs@uow.edu.au 


\title{
New informatics-based work flow paradigms in radiation oncology: the potential impact on epidemiological cancer research
}

\author{
Abstract \\ Epidemiological research is worthless without verifiable source data. Much of this data is common to the \\ clinical environment. Currently, substantial resources are allocated to data management bureaucracies in \\ attempts to ensure data accuracy. These bureaucracies developed in the era of paper records, but in the \\ present health information climate, the ability to share electronic data presents exciting possibilities, \\ while placing new responsibilities on the gatherers of information and challenging them to develop new \\ work flow paradigms. Radiation oncologists have a pivotal role to play in the processing of oncological \\ data for future epidemiological research because of the substantial overlap in data requirements.

\section{Keywords} \\ Radiation oncology, informatics, epidemiology, database management system

\section{Disciplines} \\ Medicine and Health Sciences | Social and Behavioral Sciences

\section{Publication Details} \\ Miller, A. (2005). New informatics-based work flow paradigms in radiation oncology: the potential impact \\ on epidemiological cancer research. Health Information Management Journal, 34 (3), 84-87.
}




\title{
New informatics-based work flow paradigms in radiation oncology: the potential impact on epidemiological cancer research
}

Andrew Miller

\begin{abstract}
Epidemiological research is worthless without verifiable source data. Much of this data is common to the clinical environment. Currently, substantial resources are allocated to data management bureaucracies in attempts to ensure data accuracy. These bureaucracies developed in the era of paper records, but in the present health information climate, the ability to share electronic data presents exciting possibilities, while placing new responsibilities on the gatherers of information and challenging them to develop new work flow paradigms. Radiation oncologists have a pivotal role to play in the processing of oncological data for future epidemiological research because of the substantial overlap in data requirements.
\end{abstract}

Key Words: Radiation oncology; informatics; epidemiology; database management system

\section{I ntroduction}

Radiation oncologists are medical specialists who manage cancer patients and employ radiation therapy as their main treatment modality. It is estimated that $50 \%$ of the patients who are identified in annual cancer incidence records will receive radiation therapy at some time after their diagnosis; indeed, service planning is often based on this estimate (Delaney et al. 2005).

The speed of infiltration of information technologies into radiation oncology has been astounding. So rapid has it been been that while most departments are equipped with an advanced Oncology I nformation System (OIS), few are fully aware of the improvements that can be achieved by a wider implementation that includes the radiation oncologist's work flow. Coupled with this under-appreciation is the dearth of experienced implementation services, and the reticence of oncology administrators to provide the substantial resources required to achieve meaningful implementation.

This paper describes the potential role that radiation oncologists can play in ensuring that data for epidemiological research is accurate and complete. The impact of the OIS in changing work flow will be discussed in another paper.

\section{Discussion}

\section{Current data collection paradigms}

There is an unfortunate dichotomy in the handling and status of current oncological data. In many centres a single source of data (interaction with a patient) results in dual systems of data flow. This dichotomy results from historical methods of data collection in the paper era which persist into the electronic era.

The first data flow system consists of 'normal' clinical data which is transmitted within a free text format, whether stored on paper or electronically. Similar to all such documentation, this data format is highly explicit, highly variable, largely immutable, difficult to analyse, poorly structured and unsystematised. The resultant data repository is prized as the clinical record but of little additional use.

The second data flow system consists of 'research' data which is based on the same clinical source but transmitted within a quantised format; that is, data that is classified in accordance with strictly defined categories, such as the National Cancer Institute's Common Toxicity Criteria. This format is highly implicit, invariable, highly mutable, easy to analyse, highly structured, systematised and often validated. To collect and collate this type of oncological data for research, many departments developed substantial data 'management' bureaucracies. These systems exist in parallel with and largely duplicate or parasitise the paper-based process of routine clinical data collection.

While routine data is usually recorded by clinicians and other staff in a text format, when specialised data collection is required, an additional process follows which requires that the identical data be quantised according to well recognised and validated categories (e.g., ICD-0 diagnostic codes, AJCC/UICC staging codes, NCl CTC 99 side effect criteria, RTOG/EORTC acute toxicity categories, LENT/SOMA scoring). Many of these categories are now also used in routine practice.

While the source of data is identical (the patient) and the content is identical, the 'research' collection is more structured than the 'normal' clinical data. Frequently these categorisations are scored by clinicians and then transferred to an electronic database by data managers within the bureaucracy. Moreover, it is not unknown for data managers to attempt to extract the quantised data from routine notes. Thus a single clinical interaction results in two essentially contextually identical but differently structured entries.

Difficulties in the recording of data within the two systems can be seen when a clinician might, for example, report in the clinical notes that a patient has 'mild to moderate skin reaction'. However, when entering this as trial data, the entry systems will require that the assessment of this patient's skin (as a quantised RTOG Acute Skin Reaction) be ' 1 ' (meaning faint erythema, a mild reaction) or '2' (meaning bright erythema, dry desquamation; a moderate reaction), as there is no ' 1.5 - mild to moderate' choice.

At sites where there are medical staff with some IT experience and ability, the tendency has been for them to build their own individualised but parallel electronic data repositories. These microcosms mirror the 
functionality of the data bureaucracy by being separate from the routine clinical system, often addressing an area of personal interest. These data repositories rarely influence normal data recording, or feed back to correct errors in the clinical record. (It is not unknown for departments to have all three varieties - routine clinical, data manager-based and personal data repositories!).

Unfortunately, quality assurance is usually not a prominent feature of any of these systems. In the first case, routine clinical data is rarely reviewed or changed; indeed, in some circumstances, it is considered an offence to alter data. In the second case, the degree of knowledge required to accurately assure data resides within medical staff who are largely peripheral to the operations of the bureaucracy. In the third case, the medical staff member frequently assumes that once the data is collected it is accurate because it represents a personal endeavour. The workload to compare multiple data sources with incompatible formats is largely manual and extremely difficult.

In 'research' cases, data collection is divorced from the normal stream of clinical activity and the situations where data are usually collected. Previous attempts to quality assure these data collections have found errors of over 20\% (Evans et al. 1998; Hobson, Khemani \& Singh, 2005; McCulloch, Ward \& Tekkis, 2003; Warsi, White \& McCulloch, 2002). These rates are unacceptable in data used for epidemiological purposes.

Surprisingly, modern oncology is very fortunate because of the widespread tendency to categorise most of the oncological parameters associated with patients. This tendency stems from the utility and long tradition of statistical analysis in determining what strategies are useful in the management of the cancer patient.

Oncologists therefore are aware of the importance of data and the formats used in research, but generally have been unable to develop and implement coherent strategies for data collection and verification in this format within the normal workflow.

The result is the aforementioned circumstance which conspires to duplicate and divide clinical data. From the data viewpoint, this may render the majority of data of little use for epidemiological research. From the clinical viewpoint, this may compromise the quality of patient care because the clinician might not have access to the latest and best data.

\section{Future data collection paradigms}

Data collection is a necessary process within each and every radiation oncology department. Radiation use is accompanied by a lengthy and detailed audit, with all Australian and New Zealand radiation oncology departments being required by legislation to collect and retain the details of the specifications and delivery of radiation for many years up to and after the death of the patient. The National Radiation Laboratory of New Zealand requires that a department should keep a computerised clinical database. I tem 8.2.3 states:

A suitable computerised cancer registry should be maintained that contains data on radiotherapy treatments and outcomes of at least the most common types of cancer. Treatment data should include details of the radiotherapy (target dose, fractionation system, etc) together with other treatment modalities used in combination. Outcome data should include tumour response, morbidity, mortality, and recurrence. (New Zealand National Radiation Laboratory 1992).

Similar regulatory requirements offer the prospect of routine data collection that might be provided for epidemiological research.

The recent availability of the modern OIS has promised, but not fulfilled, new opportunities for epidemiological research where the implementation of new systems within radiation oncology departments based on modern paradigms of data manipulation, data storage and retrieval will result in better validated and newer forms of data being available (Chamorro 2001).

The construct that provides the most favourable conditions for data acquisition for epidemiological research is one where all routine data collection within a department is based on the quantised data paradigm. That is, all data is regarded as and collected as if it were research data. This constitutes a reversal of most features within current data flows. To achieve this status, departments should assess software purchases intended for routine clinical use to discover whether it contains a repository with categorised data in a quantised format consistent with the type already acquired by data managers. Such a repository should cover all required fields and be expandable to include all desired fields within the areas of Record \& Verify, Schedule, Document Repository, Clinical Assessments and Disease \& Treatment outcome measures.

The modern oncology department will require that any newly purchased OIS will also be able to undertake other functionality including scheduling and billing, as well as integrate into the hospital's overall IT strategy. While the functions that enable the day-today running of the department are important, they are not the raison d'être or the prime benefit of OIS. These systems improve efficiency by virtue of their ability to integrate data, improve workflow and increase safety within the oncology context. Rather than applying resources to force radiation staff to use a generic Hospital Information System (HIS) and lose efficiency in the oncology department, effort should be expended in establishing data flow between the OIS and HIS.

The implementation of this new construct requires reorganisation based on the equally new and important concept of data ownership. The primary aims of any repository ultimately used for research should be data coverage (all items completed) and data integrity (all data makes sense). To achieve this in a normal clinical workflow requires the inclusion of the concept of ownership. This is a specifically assigned responsibility for data creation, collection and integrity within an organisation, rather than an emotional attachment to the data collection process, or a legal concept.

Once clinical data is discovered (such as the diagnosis), electronic data should be immediately entered in a quantised format by its owner. This data is then immediately available for use within the normal clinical process, and for automated quality reporting to determine whether it is complete and whether it is internally consistent. For example, it should be possible to use a database report at the end of a clinic to match 
the schedule list of follow-up visits with entries detailing patient follow-up assessments to look for entries that have not been undertaken or are incomplete. The data owner can then attend to the correction of the identified entries.

Of course the determination and assignment of data to particular groups can be difficult, as this explicit paradigm is not active in many departments and may cut across an individual's view of their professional standing and function within a system. The presence of a system of data managers tends to reinforce the view that their data is somehow different to the normal clinical data. The delegation of data collection to data managers was a pragmatic decision that occurred when there were no options for electronic records. This is not the case now and so the decision to delegate needs to be reassessed. The new OIS permits a department to reverse this delegation and redeploy data managers away from data collection and data entry towards ensuring quality and coverage, and undertaking analysis.

Nevertheless, the concept of data responsibility should be associated with frequent quality assurance of the assigned dataset, looking specifically at completion rate and data coherence, and substituting this data collection for normal clinical recording. Obviously, it would be a major undertaking to attempt to quantise all features of the clinical record, however, attempts to isolate areas where quantised data already exist and cover a major portion of an area should have early success. Treatment effects is such an area, where symptomatology is well described (e.g. $\mathrm{NCl}$ Common Toxicity Criteria).

While the proposed changes are substantial, some of the currently available OISs already possess this degree of functionality. The size of these software systems is daunting, however, and to expect busy overworked clinical radiation oncologists to undertake the process of discovery, procedural design and implementation within their work space is wishful thinking. Unfortunately, other professional groups are equally unlikely to be successful in undertaking this implementation. Professional implementation services for these systems are difficult to find, as implementation requires a high degree of knowledge about the radiation oncologist's work patterns and data use, the software design and its operational features. The result of purchasing implementation services should be a coherent system designed to deliver data of research quality while achieving similar clinical outcomes, and, it is hoped, with less effort. Implementation costs are built into other software projects (e.g., PACS introduction), however there has been no similar process considered in radiation oncology. Estimating the cost of implementation is difficult, but personal communications suggest that implementation costs are similar to or in excess of the purchase cost of the software.

Some of the methodologies required to reconcile and integrate these systems into current practice will be the subject of a later article.

There are distinct advantages in the linking of these patterns of prospective data collection within a computerised clinical system if the methodologies of collection are synchronised with the desire to reuse the data to answer research questions. Other benefits accrue from this approach. There have already been calls for retrospective practice reviews of patient outcomes to be subject to the same ethics committee approval process as prospective trials (Lertsithichai 2005). Where the quantising and storage of clinical data uses nationally approved and internationally recognised codes in a process of continuous prospective data collection to replace the usual text-based clinical record, there are no requirements for approval by ethics and professional bodies, or to ask a patient's consent to keep a record, or indeed, to even inform them of the record.

This approach is based on the fact that the generation of a clinical record is necessary in all medical situations; however, the format of such a record is not mandated by legislation. When reporting on the information within the database, confidentiality can be maintained by use of the unique database number assigned to each patient, which has no relationship to the Medical Record Number, and by the ability to include relevant patients in a report without actually opening the patient's record. In a well designed system, assiduous, frequent and early quality assurance of the entered data will negate the need to individually review charts.

Data security and storage can be integrated within a hospital's IT initiatives. Although the OIS is a separate system, its data can be freely exchanged with the HIS or any other database through common formats (e.g., NSW Department of Health Radiotherapy Information Strategy where waiting time data is reported directly from the OIS, and where a minimum dataset of diagnostic and therapy data is reported to the NSW Cancer Registry) so that the process engineering benefiting the oncology department is used while the requirement for data accumulation within the HIS is also met. The resultant system does not require the generation of paper forms except as mandated by regulation.

\section{Conclusion}

There is a surfeit of data collected within normal clinical radiation oncology using outdated text-based methods that result in repositories of largely unusable data. Implementation of a modern electronic OIS that stores data in quantised formats (e.g., NCl Common Toxicity Criteria) will enable routine clinical data to achieve a status similar to current research data.

The paradigm of data ownership with its attendant quality assurance implications can be used to construct a system which is able to ensure data coverage and integrity. Transfer of this routine data can provide epidemiological agencies with oncological data with a high degree of clinical assurance.

\section{References}

Chamorro, T. (2001). Computer-based patient record systems. Seminars in Oncological Nursing 17(1):24-33.

Delaney, G., Jacob, S., Featherstone, C. and Barton, M. (2005). The role of radiotherapy in cancer treatment: estimating optimal utilization from a review of evidence-based clinical guidelines. Cancer 104(6): 1129-1137.

Evans, W.K., Crook, J., Read, D., Morriss, J. and Logam, D.M. (1998). Capturing tumour stage in a cancer information database. Cancer Prevention and Control 2(6): 304-309. 
Hobson, J.C., Khemani, S. and Singh, A. (2005). Prospective audit of the quality of ENT emergency clinic notes before and after introduction of a computerized template. Journal of Laryngology and Otology 119(4): 264-266.

Lertsithichai, P. (2005). Waiver of consent in clinical observational research. J ournal of the Medical Association of Thailand 88(2): 275-281.

McCulloch, P., Ward, J. and Tekkis, P.P. (2003). Mortality and morbidity in gastro-oesophageal cancer surgery: initial results of ASCOT multicentre prospective cohort study. British Medical J ournal 327: 1192-1197.

New Zealand National Radiation Laboratory (1992). Code of safe practice or the use of irradiating apparatus in medical therapy. NRL C12. Available at: <http://www.nrl.moh. govt.nz/C12.pdf>. (accessed 27 July 2005).

Warsi, A.A., White, S. and McCulloch, P. (2002). Completeness of data entry in three cancer surgery databases. European J ournal of Surgical Oncology 28(8): 850-856.

Alexis Andrew Miller BSc, BMed, GradDipEd,

FRANZCR

Director, Oncology Information Services

1 Ruse Street,

Goulburn, NSW 2580

Phone (Mobile): +61 2409654239

Phone (Work): +61 248220869

Email: aamiller@goulburn.net.au 